\title{
Polarizability Matrix Extraction of a Bianisotropic Metamaterial from the Scattering Parameters of Normally Incident Plane Waves
}

\author{
Theodosios D. Karamanos, Alexandros I. Dimitriadis, and Nikolaos V. Kantartzis
}

\author{
Department of Electrical and Computer Engineering, \\ Aristotle University of Thessaloniki, Thessaloniki GR-54124, Greece \\ *corresponding author, e-mail: kant@auth.gr
}

\begin{abstract}
In this paper, a polarizability matrix retrieval method for bianisotropic metamaterials is presented. Assuming that scatterers can be modeled by electric and magnetic pointdipoles located at their centers, the induced dipole moments are analytically related to the normally incident fields, while the scattered fields are also analytically obtained for two individual cases of normal wave incidence. The latter can be combined with the incident fields so as to express the desired polarizabilities in terms of the measured or simulated scattering parameters. In this way, the polarizability matrix can be extracted by solving the resulting non-linear system of equations. The proposed technique is applied to two different split-ring resonator structures and reveals very good agreement with previously reported techniques.
\end{abstract}

\section{Introduction}

Over the past years, the problem of homogenization in metamaterials, i.e. their modeling via appropriate sets of effective parameters, has risen as a crucial subject for the sound comprehension of their properties as well as for design purposes. Two basic techniques for the calculation of effective parameters exist. The former extracts the effective constitutive parameters from the $S$-parameters of plane waves normally incident on bulk metamaterial structures, via the analytical inversion of the Fresnel-Airy equations [1], [2]. This approach has gained significant popularity due to its simplicity and adequate accuracy. However, it has also been questioned, since it completely ignores the microscopic structure of the metamaterial. Furthermore, several issues have occurred concerning the correct branch selection for the real part of the refraction index, as elaborately discussed in [3], while recently it has been proven that the constitutive parameters, so obtained, do not always represent meaningful material parameters [4]. These problems have been attributed to the bianisotropic properties of most metamaterials as well as to the effect of periodicity on the constitutive (non-local) parameters. As a result, the above mentioned methodology, although mathematically sound, produces a physically reasonable output only for anisotropic metamaterials and for structures or frequency bands with low spatial dispersion [5], [6]. The second approach, originating from the quasi-static Maxwell-Garnett mixing theory [7], employs the polarizabilities of individual scatterers in order to extract sets of effective material parameters [8], [9]. For these techniques, the knowledge of the polarizability matrix of the scatterer under study is a crucial and relatively unexplored topic.

Recently, a dynamic approach has been presented for the extraction of electric and magnetic polarizabilities of simple uniaxially anisotropic scatterers [10]. To this aim, an infinite 2-D array of scatterers is considered and their response can be modeled by electric and magnetic dipole moments, assuming only their electrically small size. Closed form expressions for the desired polarizabilities are obtained, as a function of the scattering parameters of normally incident plane waves and the interaction coefficients matrix $[C]$, whose elements have previously been calculated in the form of rapidly convergent series [11]. To the best of our knowledge, such a procedure has not been yet applied to bianisotropic scatterers, which constitute an important part of practical metamaterials. In this paper, we implement an algorithm for the extraction of the polarizability matrix, bearing in mind its common form for the most popular bianisotropic metamaterials, namely the split-ring resonators (SRRs).

\section{Polarizability extraction methodology}

In this section, our polarizability matrix extraction technique is systematically described, starting from its typical form for a well-known bianisotropic particle; the edge-coupled splitring resonator (EC-SRR). This structure can generally be modeled by three dipole moments, which can be directly estimated from the incident field and the polarizability matrix. Next, the scattered field from an array of such particles is analytically treated, by substituting the discrete distribution of scatterers with equivalent electric and magnetic currents induced on the array plane. The scattering parameters, derived from those "equivalent surfaces", can then be written as a function of these dipole moments, through their definition. The final objective is to construct and solve a system of equations in order to determine the polarizabilities through the simulated or measured S-parameters of the structure.

\subsection{Dipole moments calculation from the incident field}

Let us consider the EC-SRR of Fig. 1 as the basis of our analysis, since it is a bianisotropic particle of great importance in the literature of metamaterials. Our analysis is based on the point-dipole approximation, which requires that the dimensions of the scatterer are small enough compared to the radiation wavelength in the surrounding space (typically smaller than $\lambda / 2$ ). Under this assumption, the EC-SRR 


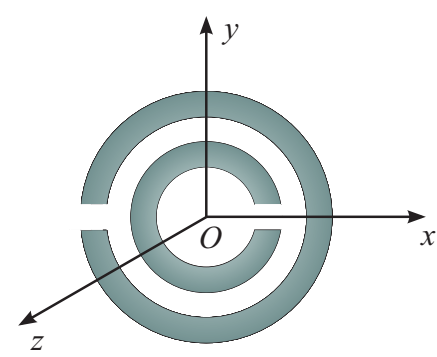

Figure 1: Geometry and axes definition of the EC-SRR.

may be appropriately modeled by two electric dipole moments $p_{x}, p_{y}$ and a magnetic dipole moment $m_{z}$ [12], which can be related to the local electric and magnetic fields at its center, $\mathbf{E}^{\text {loc }}$ and $\mathbf{H}^{\text {loc }}$, respectively, through

$$
\boldsymbol{\mu}=[\alpha] \mathbf{f}^{l o c} \Rightarrow\left[\begin{array}{c}
p_{x} \\
p_{y} \\
c^{-1} m_{z}
\end{array}\right]=\left[\begin{array}{ccc}
\alpha_{e e}^{x x} & 0 & 0 \\
0 & \alpha_{e e}^{y y} & \alpha_{e m}^{y z} \\
0 & \alpha_{m e}^{z y} & \alpha_{m m}^{z z}
\end{array}\right]\left[\begin{array}{c}
\varepsilon_{0} E_{x}^{l o c} \\
\varepsilon_{0} E_{y}^{l o c} \\
c^{-1} H_{z}^{l o c}
\end{array}\right] \text {, }
$$

where $\alpha_{e e}^{x x}, \alpha_{e e}^{y y}, \alpha_{e m}^{y z}, \alpha_{m e}^{z y}$ and $\alpha_{m m}^{z z}$ are the electric-electric, electric-magnetic, magnetic-electric and magnetic-magnetic polarizabilities of the scatterer, correspondingly. In addition, $\alpha_{e m}^{y z}=-\alpha_{m e}^{z y}$ due to the Onsager-Casimir principle [13]. As a consequence, polarizability matrix $[\alpha]$ in (1) contains four unknown quantities and thus, an equal number of linearly independent equations will be required to uniquely determine them. Considering an infinite array of particles and a plane wave normally incident on their plane, the local field at the center of any resonator is given by

$$
\mathbf{f}^{l o c}=\mathbf{f}^{i n c}+\mathbf{f}^{s c a t}=\left[\begin{array}{c}
\varepsilon_{0} E_{x}^{i n c} \\
\varepsilon_{0} E_{y}^{i n c} \\
c^{-1} H_{z}^{i n c}
\end{array}\right]+\left[\begin{array}{c}
\varepsilon_{0} E_{x}^{s c a t} \\
\varepsilon_{0} E_{y}^{s c a t} \\
c^{-1} H_{z}^{s c a t}
\end{array}\right],
$$

where $\mathbf{f}^{\text {inc }}$ is the incident field and $\mathbf{f}^{\text {scat }}$ stands for the scattered field at the center of an arbitrary scatterer produced from its neighboring ones, defined by

$$
\mathbf{f}^{s c a t}=[C] \boldsymbol{\mu} \Rightarrow\left[\begin{array}{c}
\varepsilon_{0} E_{x}^{s c a t} \\
\varepsilon_{0} E_{y}^{s c a t} \\
c^{-1} H_{z}^{s c a t}
\end{array}\right]=\left[\begin{array}{ccc}
C_{e e}^{x x} & 0 & 0 \\
0 & C_{e e}^{y y} & C_{e m}^{y z} \\
0 & C_{e m}^{y z} & C_{m m}^{z z}
\end{array}\right]\left[\begin{array}{c}
p_{x} \\
p_{y} \\
c^{-1} m_{z}
\end{array}\right] .
$$

The components of the interaction coefficient matrix $[C]$ are computed via the dyadic Green's function method [11]. By substituting (2) and (3) into (1), the dipole moments of an arbitrary scatterer can be expressed as

$$
\boldsymbol{\mu}=\left([\alpha]^{-1}-[C]\right)^{-1} \mathbf{f}^{i n c} .
$$

Let us, now, consider two different cases of normal incidence to an infinite array of EC-SRRs (in the following, we will refer to these arrays with the term "metasurface"). The first metasurface is depicted in Fig. 2. For this case, the components of the incident plane wave are

$$
\mathbf{f}_{A}^{i n c}=\left[\begin{array}{llll}
\varepsilon_{0} E_{x, A}^{i n c} & \varepsilon_{0} E_{y, A}^{i n c} & c^{-1} H_{z, A}^{i n c}
\end{array}\right]^{T}=\left[\begin{array}{llll}
0 & \varepsilon_{0} E_{y, A}^{i n c} & \varepsilon_{0} & E_{y, A}^{i n c}
\end{array}\right]^{T}
$$

or, by normalizing the incident electric field amplitude,

$$
\mathbf{f}_{A}^{i n c}=\left[\begin{array}{lll}
0 & \varepsilon_{0} & \varepsilon_{0}
\end{array}\right]^{T} \text {. }
$$

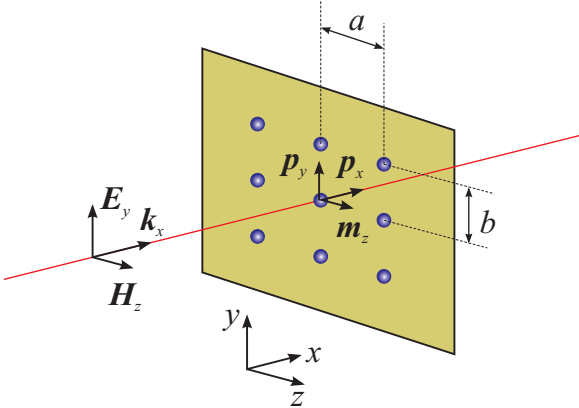

Figure 2: The first metasurface of bianisotropic scatterers at the $x=0$ plane. The periods of the lattice along the $y$ - and $z$ direction, are $b$ and $a$, respectively. A TEM wave propagating along the $x$-axis impinges normally on the metasurface.

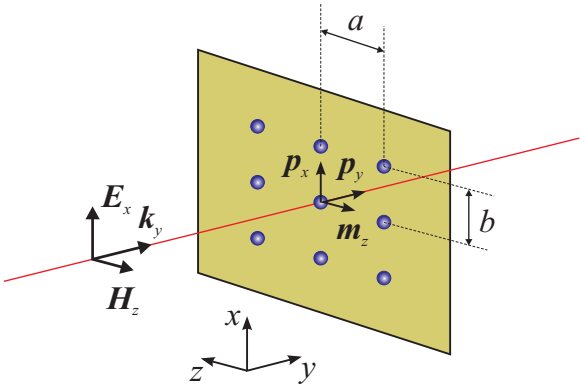

Figure 3: The second metasurface of bianisotropic scatterers at the $y=0$ plane. The periods of the lattice along the $x$ - and $z-$, direction are $b$ and $a$, respectively. A TEM wave propagating along the $y$-axis impinges normally on the metasurface.

Substituting (5b) into (4), we acquire a set of dipole moments, denoted as

$$
\boldsymbol{\mu}_{A}=\left[\begin{array}{lll}
p_{x, A} & p_{y, A} & c^{-1} m_{z, A}
\end{array}\right]^{T},
$$

as a function of the polarizability matrix $[\alpha]$.

On the other hand, the second metasurface is given in Fig. 3. The components of the incident plane wave are

$$
\mathbf{f}_{B}^{i n c}=\left[\begin{array}{lll}
\varepsilon_{0} E_{x, B}^{i n c} & \varepsilon_{0} E_{y, B}^{i n c} & c^{-1} H_{z, B}^{i n c}
\end{array}\right]^{T}=\left[\begin{array}{llll}
\varepsilon_{0} E_{x, B}^{i n c} & 0 & -\varepsilon_{0} E_{x, B}^{i n c}
\end{array}\right]^{T}
$$

or by normalizing the incident electric field amplitude,

$$
\mathbf{f}_{B}^{i n c}=\left[\begin{array}{lll}
\varepsilon_{0} & 0 & -\varepsilon_{0}
\end{array}\right]^{T} .
$$

Plugging (6b) into (4), a second set of dipoles with respect to the polarizabilities of (1) is obtained, which is defined by

$$
\boldsymbol{\mu}_{B}=\left[\begin{array}{lll}
p_{x, B} & p_{y, B} & c^{-1} m_{z, B}
\end{array}\right]^{T} .
$$

\subsection{Dipole moments relation to the $S$-parameters}

Having obtained the dipole moments of the scatterers from the incident field, we can, now, express them as a function of the simulated or measured scattering parameters of an equivalent homogenized surface.

Let us recall the two metasurfaces of Figs 2 and 3. For the first incidence case, the discrete array of electric and magnetic dipoles can be substituted with equivalent surface electric and magnetic polarizations, given by

$$
P_{s x, A}=p_{x, A} \sum_{m=-\infty}^{\infty} \sum_{n=-\infty}^{\infty} \delta(y-m a) \delta(z-n b) .
$$


Utilizing the Poisson summation formula, (7) transforms to

$$
P_{s x, A}=\frac{p_{x, A}}{a b} \sum_{m=-\infty}^{\infty} \sum_{n=-\infty}^{\infty} e^{j\left(\frac{2 \pi m}{a} y+\frac{2 \pi n}{b} z\right)} .
$$

In a similar fashion, electric polarization $P_{s y, A}$ and magnetic polarization $M_{s z, A}$ are found equal to

$$
\begin{gathered}
P_{s y, A}=\frac{p_{y, A}}{a b} \sum_{m=-\infty}^{\infty} \sum_{n=-\infty}^{\infty} e^{j\left(\frac{2 \pi m}{a} y+\frac{2 \pi n}{b} z\right)}, \\
M_{s z, A}=\frac{m_{z, A}}{a b} \sum_{m=-\infty}^{\infty} \sum_{n=-\infty}^{\infty} e^{j\left(\frac{2 \pi m}{a} y+\frac{2 \pi n}{b} z\right)},
\end{gathered}
$$

where $p_{x, A}, p_{y, A}$, and $m_{z, A}$, are the dipole moments of an arbitrary resonator, as described in subsection 2.1.

Following [14], only the first (zeroth-order) term of the sums in (8) represents a propagating wave, while all higherorder terms correspond to evanescent waves. Therefore, in the far-field region, the discrete array can be seen as an equivalent surface polarizations/magnetization

$$
P_{s x, A}=\frac{p_{x, A}}{a b}, \quad P_{s y, A}=\frac{p_{y, A}}{a b}, \quad M_{s z, A}=\frac{m_{z, A}}{a b} .
$$

Likewise, the surface electric and magnetic polarizations for the second metasurface are given by

$$
\begin{gathered}
P_{s x, B}=\frac{p_{x, B}}{a b} \sum_{m=-\infty}^{\infty} \sum_{n=-\infty}^{\infty} e^{j\left(\frac{2 \pi m}{a} x+\frac{2 \pi n}{b} z\right)}, \\
P_{s y, B}=\frac{p_{y, B}}{a b} \sum_{m=-\infty}^{\infty} \sum_{n=-\infty}^{\infty} e^{j\left(\frac{2 \pi m}{a} x+\frac{2 \pi n}{b} z\right)}, \\
M_{s z, B}=\frac{m_{z, B}}{a b} \sum_{m=-\infty}^{\infty} \sum_{n=-\infty}^{\infty} e^{j\left(\frac{2 \pi m}{a} x+\frac{2 \pi n}{b} z\right)},
\end{gathered}
$$

and, similar to (9), the polarization terms that contribute in the far-field are expressed as

$$
P_{s x, B}=\frac{p_{x, B}}{a b}, \quad P_{s y, B}=\frac{p_{y, B}}{a b}, \quad M_{s z, B}=\frac{m_{z, B}}{a b} .
$$

The scattered field from equivalent homogenized surfaces can be evaluated through boundary conditions [15], [16]

$$
\begin{gathered}
\hat{\mathbf{n}} \times\left(\left.\mathbf{H}\right|_{z=0^{-}}-\left.\mathbf{H}\right|_{z=0^{+}}\right)=\partial_{t} \mathbf{P}_{s}+\nabla \times \mathbf{M}_{s}=\mathbf{J}_{s}, \\
\hat{\mathbf{n}} \times\left(\left.\mathbf{E}\right|_{z=0^{-}}-\left.\mathbf{E}\right|_{z=0^{+}}\right)=\partial_{t} \mathbf{M}_{s}+\nabla \times \mathbf{P}_{s}=-\mathbf{K}_{s} .
\end{gathered}
$$

Applying the right parts of (12) and (13), for the polarizations of (9), we acquire the equivalent electric, $\mathbf{J}_{s}$, and magnetic, $\mathbf{K}_{s}$, surface currents

$$
\begin{gathered}
\mathbf{J}_{s}=j \omega \frac{p_{x}}{a b} \hat{\mathbf{x}}+j \omega \frac{p_{y}}{a b} \hat{\mathbf{y}}, \\
\mathbf{K}_{s}=j \omega \mu_{0} \frac{m_{z}}{a b} \hat{\mathbf{z}} .
\end{gathered}
$$

Furthermore, equating these results to the left-hand sides of (12) and (13), the scattering field from the equivalent homogenized metasurface is derived. For the first case, the scattered fields are obtained as

$$
\hat{\mathbf{x}} \times\left(\left.\mathbf{H}\right|_{x=0^{-}}-\left.\mathbf{H}\right|_{x=0^{+}}\right)=\mathbf{J}_{s} \Rightarrow\left(\left.H_{z}\right|_{x=0^{+}}-\left.H_{z}\right|_{x=0^{-}}\right)=J_{s y} .
$$

As expected, surface current components normal to the surface do not produce any scattered field. Considering also that

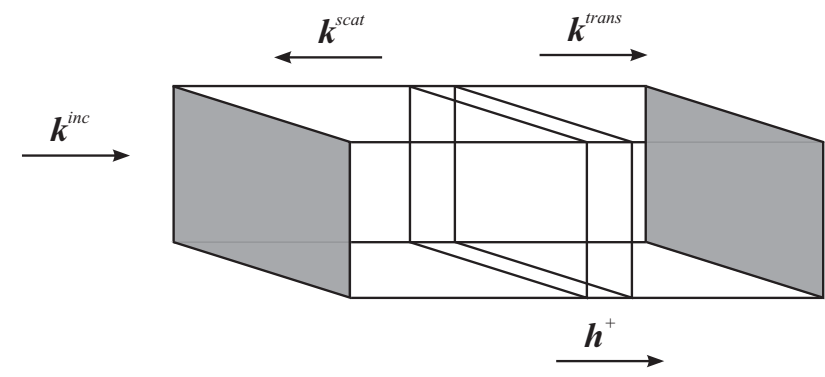

Figure 4: The simulation/measurement configuration. Propagation occurs along the positive $h$-direction, which corresponds to $x$ - and $y$-axis (first and second case, respectively). The central rectangle depicts the metasurface and gray areas the reference planes, at a distance of $\pm l$ for the metasurface.

$$
\left.H_{z}\right|_{x=0^{+}} ^{\text {scat }, A}=-\left.H_{z}\right|_{x=0^{-}} ^{\text {scat } A} \quad \text { and }\left.\quad E_{y}\right|_{x=0^{+}} ^{\text {scat }, A}=\left.Z_{0} H_{z}\right|_{x=0^{+}} ^{\text {scat }, A},
$$

equation (16) results in

$$
\mathbf{E}_{J_{s}, A}^{s c a t}=-j \omega \frac{Z_{0} p_{y, A}}{2 a b} e^{-j k_{0}|x|} \hat{\mathbf{y}},
$$

where $Z_{0}$ is the characteristic impedance in vacuum. Similarly, from (13) and (15), the scattered electric field component due to the magnetic surface current, is given by

$$
\mathbf{E}_{K_{s}, A}^{s c a t}=-\operatorname{sgn}(x) \frac{j \omega \mu_{0} m_{z, A}}{2 a b} e^{-j k_{0}|x|} \hat{\mathbf{y}} .
$$

Summation of (17) and (18) leads to the scattered field expression of the first equivalent surface, i.e.

$$
\mathbf{E}_{A}^{s c a t}=-\frac{j \omega}{2 a b}\left(Z_{0} p_{y, A}+\operatorname{sgn}(x) \mu_{0} m_{z, A}\right) e^{-j k_{0}|x|} \hat{\mathbf{y}},
$$

whereas the scattered field for the second one is derived by

$$
\mathbf{E}_{B}^{s c a t}=-\frac{j \omega}{2 a b}\left(Z_{0} p_{x, B}+\operatorname{sgn}(y) \mu_{0} m_{z, B}\right) e^{-j k_{0}|y|} \hat{\mathbf{x}} .
$$

Through (19) and (20), we may find the $S$-parameters for each equivalent metasurface, owing to their relation with the reflected and transmitted wave at the reference planes $\pm l$, as presented in Fig. 4. Distance $l$ is considered to be sufficiently large so that the higher order terms of (8) and (10) are adequately attenuated.

Subsequently, considering the fields on the reference planes, we calculate $S$-parameters by means of

$$
\begin{gathered}
S_{11}=\frac{E^{\text {scat }}(h=-l)}{E^{\text {inc }}(h=-l)}=\frac{E^{\text {scat }}}{E^{\text {inc }}} e^{-j 2 k_{0} l}, \\
S_{21}=\frac{E^{\text {trans }}(h=+l)}{E^{\text {inc }}(h=-l)}=\frac{E^{i n c}+E^{\text {scat }}}{E^{\text {inc }}} e^{-j 2 k_{0} l} .
\end{gathered}
$$

Substitution of (19) and (20) in (21) and (22), finally, yields the dipole moments parallel to each equivalent surface as a function of the $S$-parameters

$$
\begin{aligned}
p_{y, A} & =\frac{j a b}{\omega Z_{0}}\left(S_{11, A} e^{j 2 k_{0} l}+S_{21, A} e^{j 2 k_{0} l}-1\right), \\
c^{-1} m_{z, A} & =-\frac{j a b}{\omega Z_{0}}\left(S_{11, A} e^{j 2 k_{0} l}-S_{21, A} e^{j 2 k_{0} l}+1\right), \\
p_{x, B} & =\frac{j a b}{\omega Z_{0}}\left(S_{11, B} e^{j 2 k_{0} l}+S_{21, B} e^{j 2 k_{0} l}-1\right),
\end{aligned}
$$




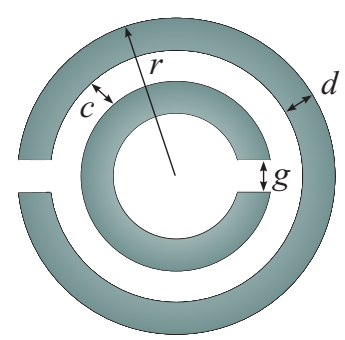

Figure 5: Geometry of the simulated EC-SRR, with $r=3.2$ $\mathrm{mm}, c=0.5 \mathrm{~mm}, d=0.5 \mathrm{~mm}$, and $g=0.5 \mathrm{~mm}$. The periods of the lattice, as depicted in Figs 2 and 3, are $a=b=10 \mathrm{~mm}$, and the distance from the reference planes is $l= \pm 3 a$.

$$
c^{-1} m_{z, B}=-\frac{j a b}{\omega Z_{0}}\left(S_{11, B} e^{j 2 k_{0} l}-S_{21, B} e^{j 2 k_{0} l}+1\right) .
$$

If we plug the dipole moments, evaluated from (4) for both incidence cases, into (23), a system of four linearly independent equations is obtained, containing polarizabilities $\alpha_{e e}^{x x}, \alpha_{e e}^{y y}, \alpha_{e m}^{y z}$, and $\alpha_{m m}^{z z}$. Moreover, due to (1), equation (23c) contains only the $a_{e e}^{x x}$ term and hence it can be solved directly, thus reducing the final system to three equations for the unknown polarizabilities $\alpha_{e e}^{y y}, \alpha_{e m}^{y z}$, and $\alpha_{m m}^{z z}$. Such system is non-linear and a closed-form solution is not generally feasible. However, in the special case of $\alpha_{e m}^{y z}=0$ the closedform solution of (23) is viable and the result coincides with the method presented in [10], as verified in Section 3. In the following section, we apply the Newton-Raphson method (see Appendix I) to numerically solve (23).

\section{Results and Discussion}

The proposed polarizability extraction methodology is, herein, applied to two SRR structures. All simulations are conducted via the frequency domain solver of the CST MWS ${ }^{\mathrm{TM}}$ computational package [17]. The first structure is the bianisotropic EC-SRR of Fig. 5, which is known to produce a strong magnetic field, opposite to the incident and perpendicular to its plane, on its first resonance frequency. The transmission coefficient of EC-SRR for $x$ - and $y$-incidence is displayed in Fig. 6, indicating the first two resonances. The real part of the polarizabilitites, extracted by our technique, is given in Fig. 7. It is straightforward to detect that both $\alpha_{e e}^{y y}$ and $\alpha_{m m}^{z z}$ become resonant approximately at the same frequency. This outcome is in agreement with the assumptions of [12]. Next, the $\alpha_{e e}^{x x}, \alpha_{e e}^{y y}$, and $\alpha_{m m}^{z z}$ polarizabilities are further validated by comparing them with the SipeKranendonk condition [18] for lossless anisotropic scatterers, which reads (for $i=x, y, z$ and $j=e, m$ )

$$
\operatorname{Im}\left\{\frac{1}{\alpha_{j j}^{i i}}\right\}=\frac{k_{0}^{3}}{6 \pi} .
$$

As presented in Fig. 8, outside the resonance bands, where (24) is not accurate due to the presence of bianisotropic effects, the Sipe-Kranendonk condition is satisfied.

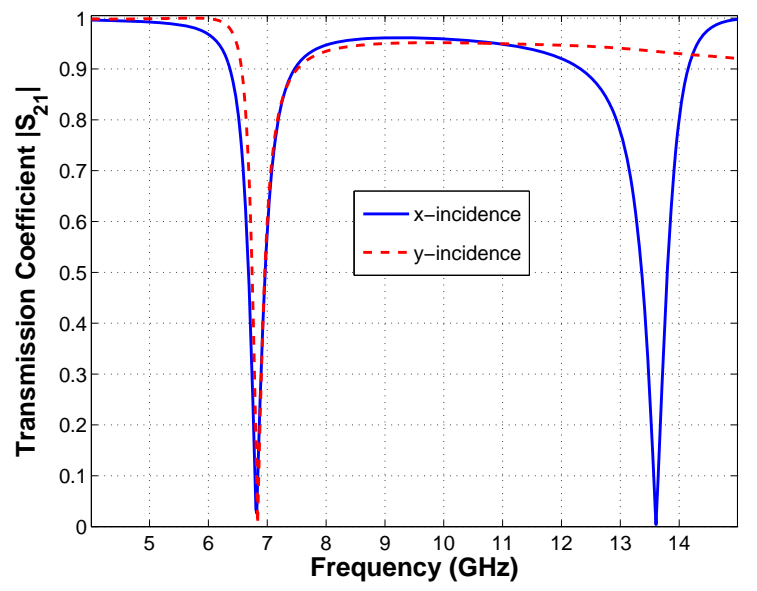

Figure 6: Transmission coefficient for the EC-SRR of Fig. 6, for the $x$ - and $y$-incidence, respectively. The axes convention is the same as that established in Fig. 1.

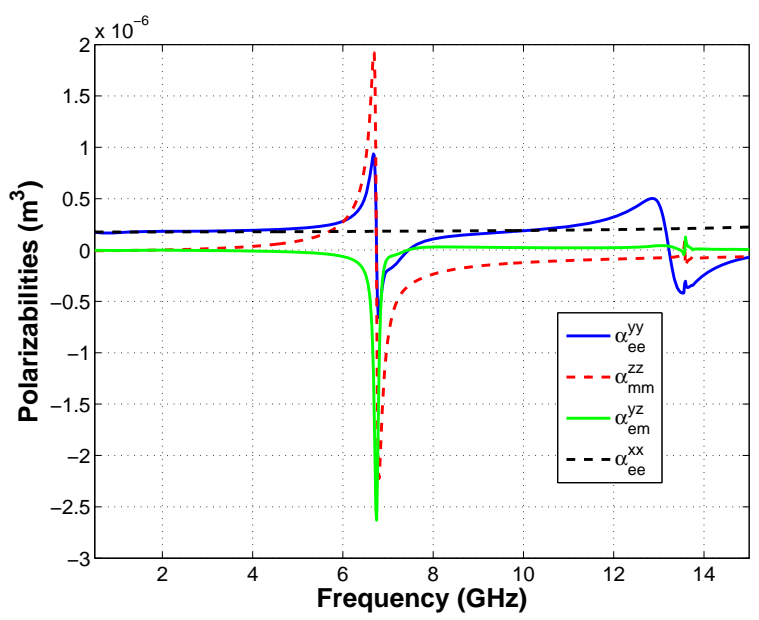

Figure 7: Real part of the polarizabilities of the EC-SRR, extracted via the proposed method.

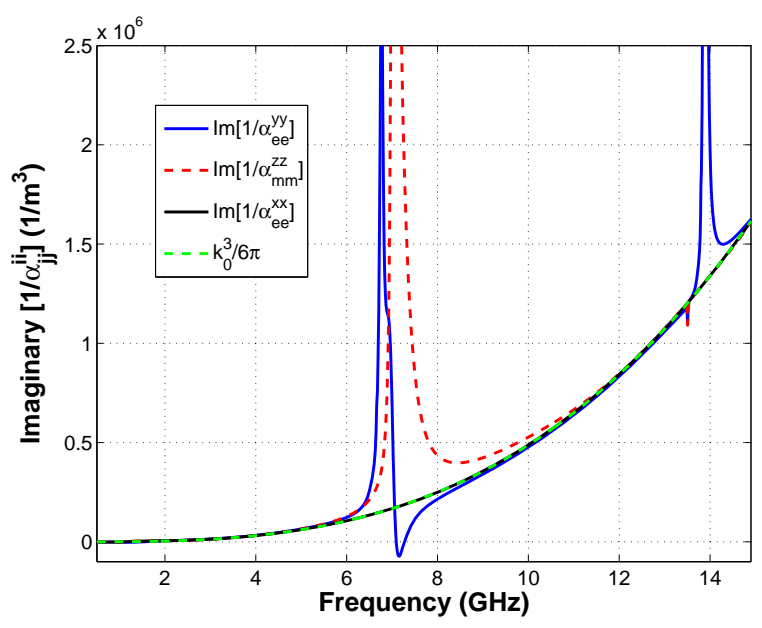

Figure 8: Comparison of the Sipe-Kranendonk criterion for lossless scatterers with the polarizabilities of the EC-SRR extracted in Fig. 7. The criterion is not satisfied on resonance bands, where the anisotropic assumption is not valid. 


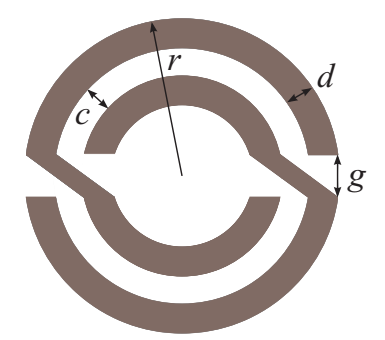

Figure 9: Geometry of the simulated NB-SRR, with $r=3.7$ $\mathrm{mm}, c=0.5 \mathrm{~mm}, d=0.5 \mathrm{~mm}$, and $g=0.5 \mathrm{~mm}$. The periods of the lattice, as depicted in Figs 2 and 3, are $a=b=15 \mathrm{~mm}$, and the distance from the reference planes is $l= \pm 3 a$.

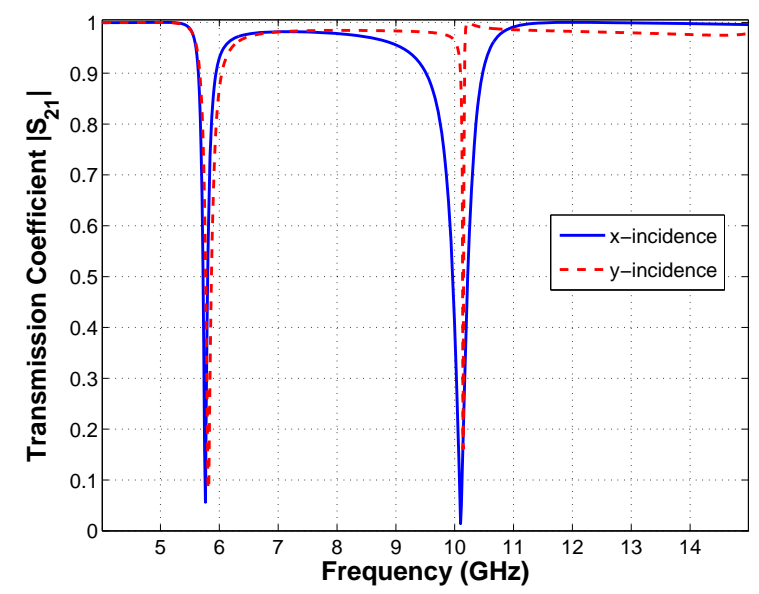

Figure 10: Transmission coefficient for the NB-SRR of Fig. 9 , for the $x$ - and $y$-incidence, respectively. The axes convention is the same as that established in Fig. 1.

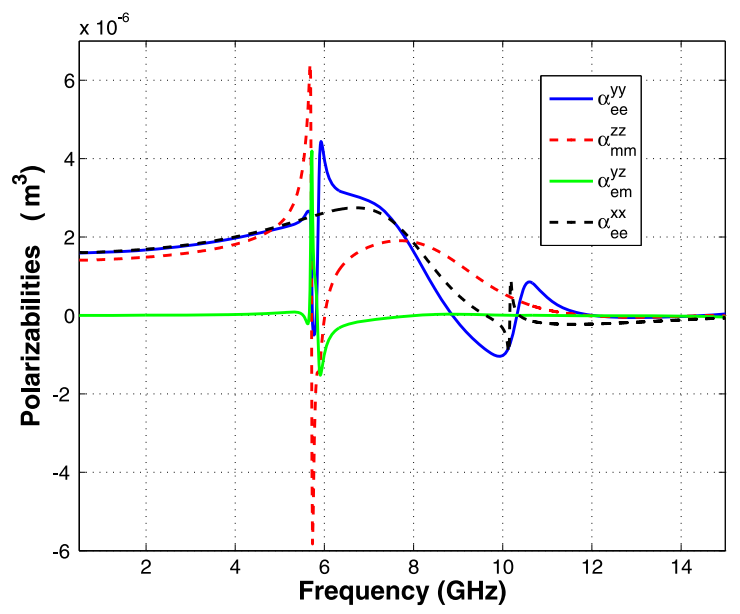

Figure 11: Real part of the polarizabilities of the NB-SRR, extracted via the proposed method.

Next, let us focus on the non-bianisotropic SRR (NB-SRR) of Fig. 9. Despite its designation, bianisotropic effects are not totally absent in that structure, as our analysis will reveal. The transmission coefficient for the two first resonances is illustrated in Fig. 10 for both $x$ - and $y$-incidence, while the real parts of the corresponding polarizabilities are presented in Fig. 11. Figure 12 presents a comparison of our results with those of [10], valid for uniaxially anisotropic scatterers,

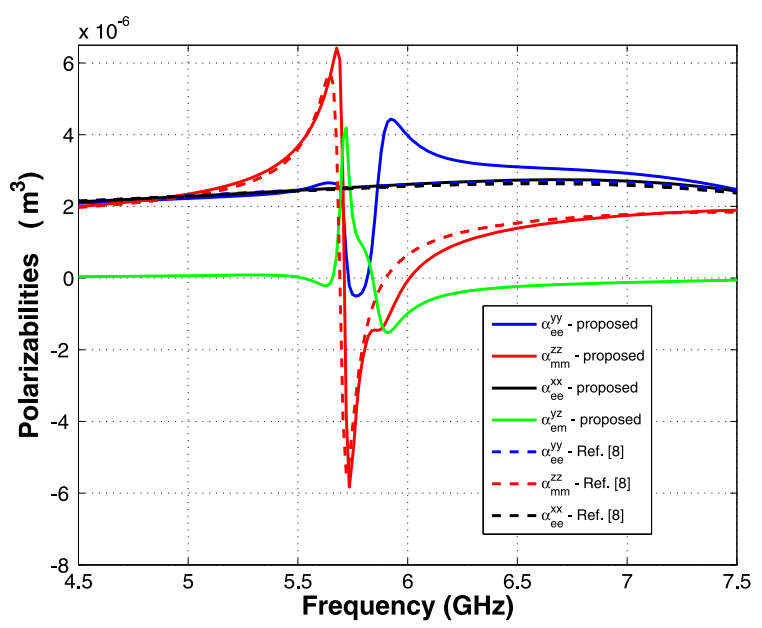

Figure 12: Detail of Fig. 11, including comparison of the results with those of [10].

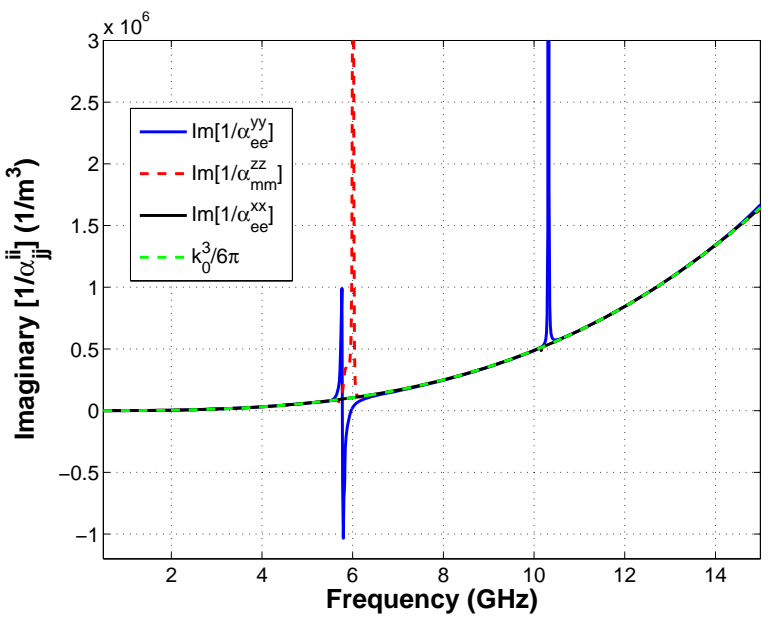

Figure 13: Comparison of the Sipe-Kranendonk criterion for lossless scatterers with the polarizabilities of the NB-SRR extracted in Fig. 11. The criterion is not satisfied on resonance bands, where the anisotropic assumption is not valid.

where a very good agreement can be promptly detected. Deviations are significant only in the resonance bands, where a considerable electric-magnetic polarizability has been found in our simulations, which has not been taken into account in [10]. These deductions suggest that the proposed technique can be regarded as a generalization of [10], as already mentioned before. Finally in Fig. 13, by applying the Sipe-Kranendonk criterion (24) on $\alpha_{e e}^{x x}, \alpha_{e e}^{y y}$, and $\alpha_{m m}^{z z}$, we attain additional verification on the efficiency of our method.

\section{Conclusions}

A novel methodology for extracting polarizabilities from bianisotropic scatterers has been introduced in this paper. Its formulation is based on the use of the point-dipole approximation for both the microscopic and macroscopic description of two equivalent metasurfaces. Initially, the dipole moments are acquired as a function of the polarizabilities of the scatterers. Assuming, additionally, that infinite rectangular arrays of scatterers constitute equivalent homogenized 
metasurfaces, the equivalent surface currents generated by a normally incident wave are analytically obtained and dipole moments are expressed as a function of the simulated/ measured $S$-parameters. Furthermore, equating the polarizabilities, acquired from the microscopic and macroscopic approach, a non-linear system of equations for the unknown polarizabilities is derived, which can be numerically solved.

Concerning the certification of our algorithm, two of the most popular SRR structures, namely the EC-SRR and NBSRR, have been investigated. The results are in very good agreement with those reported already in the relevant literature and have been further validated by means of the SipeKranendonk criterion. Moreover, for the case of the NBSRR, outcomes have been compared with those of an existing method, developed for uniaxially anisotropic scatterers. Again, our results coincidence is very satisfactory, while some differences between the two schemes is attributed to the existence of a non-vanishing electric-magnetic polarizability inside the resonance band, which is a priori ignored in [10].

Future work is going to address the expansion of the reported methodology to a broader set of scatterers, with an even larger number of unknown polarizabilites, on condition that an equal number of different and independent measurements can be carried out. Finally, polarizabilities extracted through the method could be utilized in rigorous homogenization techniques that are currently being developed, such as the one developed in [4].

\section{Appendix I}

\section{The Newton-Raphson method for non-linear systems}

Assume a non-linear system

$$
[\mathbf{A}(u)]\{\mathbf{u}\}=\{\mathbf{b}\},
$$

or in full form

$$
\begin{aligned}
& a_{11} u_{1}+a_{12} u_{2}+\ldots+a_{1 n} u_{n}=b_{1} \\
& a_{21} u_{1}+a_{22} u_{2}+\ldots+a_{2 n} u_{n}=b_{2} \\
& \ldots \ldots \ldots \ldots \ldots \ldots \ldots \ldots \ldots \ldots \ldots \ldots \ldots \ldots \ldots \ldots \ldots \ldots \ldots \ldots \ldots \ldots \ldots \ldots \ldots \ldots \\
& a_{n 1} u_{1}+a_{n 2} u_{2}+\ldots+a_{n n} u_{n}=b_{n}
\end{aligned}
$$

where coefficients $a_{i j}$, are not constant, but functions of variables $u_{i}$. Vector $\{\mathbf{u}\}^{T}=\left\{u_{1}, u_{2}, \ldots, u_{n}\right\}$ contains the unknown parameters and $\{\mathbf{b}\}^{T}=\left\{b_{1}, b_{2}, \ldots, b_{n}\right\}$ contains constant components. Moreover, system (25) can be written as

$$
\mathbf{f}=\left[\begin{array}{c}
f_{1} \\
f_{2} \\
\ldots \\
f_{n}
\end{array}\right]=\left[\begin{array}{c}
0 \\
0 \\
\ldots \\
0
\end{array}\right],
$$

where $f_{i}=a_{i 1} u_{1}+a_{i 2} u_{2}+\cdots+a_{i n} u_{n}-b_{i}=0$ for $i=(1,2, \ldots, n)$.

According to the Newton-Raphson method, the approximate solutions $u^{(k+1)}, u^{(k+1)}$ on the $(k+1)$-th and $k$-th step respectively, are given by

$$
\mathbf{u}^{(k+1)}=\mathbf{u}^{(k)}+\delta \mathbf{u}^{(k)},
$$

where vector $\delta \mathbf{u}^{(k)}$ represents the components' modification, while advancing from the $k$-th to the $(k+1)$-th step.
Let us consider the following Taylor expansion, where second order terms are forfeit

$$
\begin{aligned}
f_{i}\left(u_{1}^{(k+1)}, u_{2}^{(k+1)}, \ldots, u_{n}^{(k+1)}\right) & =f_{i}\left(u_{1}^{(k)}, u_{2}^{(k)}, \ldots, u_{n}^{(k)}\right) \\
& +\cdots+\frac{\partial f_{i}}{\partial u_{1}} \delta u_{1}^{(k)}+\frac{\partial f_{i}}{\partial u_{2}} \delta u_{2}^{(k)}+\cdots+\frac{\partial f_{i}}{\partial u_{n}} \delta u_{n}^{(k)},
\end{aligned}
$$

for $i=(1,2, \ldots, n)$.

Setting $f_{i}\left(u_{1}^{(k+1)}, u_{2}^{(k+1)}, \ldots, u_{n}^{(k+1)}\right)=0$, we extract the following system of equations

$$
\begin{aligned}
& \frac{\partial f_{1}}{\partial u_{1}} \delta u_{1}^{(k)}+\frac{\partial f_{1}}{\partial u_{2}} \delta u_{2}^{(k)}+\cdots+\frac{\partial f_{1}}{\partial u_{n}} \delta u_{n}^{(k)}=-f_{1}\left(u_{1}^{(k)}, u_{2}^{(k)}, \ldots, u_{n}^{(k)}\right) \\
& \frac{\partial f_{2}}{\partial u_{1}} \delta u_{1}^{(k)}+\frac{\partial f_{2}}{\partial u_{2}} \delta u_{2}^{(k)}+\cdots+\frac{\partial f_{2}}{\partial u_{n}} \delta u_{n}^{(k)}=-f_{2}\left(u_{1}^{(k)}, u_{2}^{(k)}, \ldots, u_{n}^{(k)}\right) \\
& \frac{\partial f_{n}}{\partial u_{1}} \delta u_{1}^{(k)}+\frac{\partial f_{n}}{\partial u_{2}} \delta u_{2}^{(k)}+\cdots+\frac{\partial f_{n}}{\partial u_{n}} \delta u_{n}^{(k)}=-f_{n}\left(u_{1}^{(k)}, u_{2}^{(k)}, \ldots, u_{n}^{(k)}\right)
\end{aligned}
$$

Using the subsequent Jacobian matrix

$$
\mathbf{J}=\left[\begin{array}{cccc}
\frac{\partial f_{1}}{\partial u_{1}} & \frac{\partial f_{1}}{\partial u_{2}} & \ldots & \frac{\partial f_{1}}{\partial u_{n}} \\
\frac{\partial f_{2}}{\partial u_{1}} & \frac{\partial f_{2}}{\partial u_{2}} & \ldots & \frac{\partial f_{2}}{\partial u_{n}} \\
\cdots & \ldots & \ldots & \ldots \\
\frac{\partial f_{n}}{\partial u_{1}} & \frac{\partial f_{n}}{\partial u_{2}} & \ldots & \frac{\partial f_{n}}{\partial u_{n}}
\end{array}\right],
$$

equations (29) are rewritten as

$$
\mathbf{J} \delta \mathbf{u}^{(k)}=-\mathbf{f}\left(\mathbf{u}^{(k)}\right) .
$$

Substituting (31) in (27) and taking into account (26), we derive

$$
\mathbf{u}^{(k+1)}=\mathbf{u}^{(k)}-\mathbf{J}^{-1}\left[\mathbf{A}\left(\mathbf{u}^{(k)}\right) \mathbf{u}^{(k)}-\mathbf{b}\right],
$$

where the elements of $\mathbf{J}$ are calculated via

$$
J_{i j}=\frac{\partial f_{i}}{\partial u_{i}}=a_{i j}+\sum_{k=1}^{n} \frac{\partial a_{i k}}{\partial u_{i}} u_{k} .
$$

\section{Acknowledgements}

This research has been co-financed by the EU (European Social Fund - ESF) and Greek national funds through the Operational Program "Education and Lifelong Learning" of the National Strategic Reference Framework (NSRF) - Research Funding Program: ARISTEIA I. Investing in knowledge society through the European Social Fund.

\section{References}

[1] D.R. Smith, S. Schultz, P. Markoš, C.M. Soukoulis, Determination of effective permittivity and permeability of metamaterials from reflection and transmission coefficients, Phys. Rev. B 65: pp. 195104(1-5), 2002.

[2] X. Chen, T.M. Grzegorczyk, B.-I. Wu, J. Pacheco, J.A. Kong, Robust method to retrieve the constitutive effective parameters of metamaterials, Phys. Rev. E 70: pp. 
016608(1-7), 2004.

[3] V.V. Varadan, R. Ruyen, Unique retrieval of complex permittivity and permeability of dispersive materials from reflection and transmitted fields by enforcing causality, IEEE Trans. Microw. Theory and Tech. 55: pp. 2224-2230, 2007.

[4] A. Alù, First-principles homogenization theory for periodic metamaterials, Phys. Rev. B 84: pp. 075153(118), 2011.

[5] T. Koschny, P. Markoš, D.R. Smith, C.M. Soukoulis, Resonant and antiresonant frequency dependence of the effective parameters of metamaterials, Phys. Rev. E 68: p. 065602(R)(1-4), 2003.

[6] T. Koschny, P. Markoš, D.R. Smith, C.M. Soukoulis, Reply to comments on "Resonant and antiresonant frequency dependence of the effective parameters of metamaterials", Phys. Rev. E 70: p. 048603, 2004.

[7] A.H Sihvola, Electromagnetic Mixing Formulas and Applications, IEEE Electromagnetic Wave Series, Piscataway, New Jersey, 2000.

[8] D. Morits, C.R. Simovski, Electromagnetic characterization of planar and bulk metamaterials: A theoretical study, Phys. Rev. B 82: pp. 165114(1-10), 2010.

[9] A.I. Dimitriadis, N.V. Kantartzis, I.T. Rekanos, T.D. Tsiboukis, Efficient metafilm/metasurface characterization for obliquely incident TE waves via surface susceptibility models, IEEE Trans. Magn. 48: pp. 367370, 2012.

[10] A.D. Scher, E.F. Kuester, Extracting the bulk effective parameters of a metamaterial via the scattering from a single planar array of particles, Metamaterials 3: pp. 44-55, 2009.

[11] P.A. Belov, C.R. Simovski, Homogenization of electromagnetic crystals formed by uniaxial resonant scatterers, Phys. Rev. E 72: pp. 026615(1-15), 2005.

[12] R. Marqués, F. Martín, M. Sorolla, Metamaterials with negative parameters: Theory, design and microwave applications, John Wiley \& Sons, New York, 2008.

[13] A. Serdyukov, I. Semchenko, S. Tretyakov, A. Sihvola, Electromagnetics of bi-anisotropic materials: Theory and applications, Gordon and Breach Science Publishers, Amsterdam, 2001.

[14] A.I. Dimitriadis, D. Sounas, N.V. Kantartzis, C. Caloz, T.D. Tsiboukis, Surface susceptibility bianisotropic matrix model for periodic metasurfaces of uniaxially mono-anisotropic scatterers under oblique TE-wave incidence, IEEE Trans. Antennas Propag., 2012 (to appear).

[15] R.E. Collin, Field theory of guided waves, IEEE Press, New Jersey, 1990.

[16] C.L. Holloway, M.A. Mohamed, E.F. Kuester, A. Dienstfrey, Reflection and transmission properties of a metafilm: with an application to a controllable surface composed of resonant particles, IEEE Trans. Electromagn. Compat. 47: pp. 853-865, 2005.
[17] CST MWS ${ }^{\mathrm{TM}}$ : Computer Simulation Technology: Microwave Studio, Computer Simulation Technology Std., 2011.

[18] S.A. Tretyakov, Analytical modeling in applied electromagnetics, Arthech House Publishers, Boston, pp. 96-98, 2003. 